\title{
Intranasal corticosteroids do not affect intraocular pressure or lens opacity: a systematic review of controlled trials*
}

\author{
Nazanin Ahmadi', Kornkiat Snidvongs ${ }^{4}$, Larry Kalish ${ }^{3}$, Raymond Sacks ${ }^{2,3}$, \\ Krishna Tumuluri ${ }^{5}$, Geoff Wilcsek6, Richard Harvey ${ }^{1,2}$ \\ ' Rhinology and Skull Base Applied Medical Research Centre, University of New South Wales, Sydney, NSW, Australia \\ ${ }^{2}$ Australian School of Advanced Medicine, Macquarie University, Sydney, NSW, Australia \\ ${ }^{3}$ Otolaryngology and Head and Neck Surgery, Concord Hospital and University of Sydney, Sydney, NSW, Australia \\ ${ }^{4}$ Department of Otolaryngology, Faculty of Medicine, Chulalongkorn University, Bangkok, Thailand \\ ${ }^{5}$ Department of Ophthalmology Westmead Adult and Children's Hospitals, Liverpool Hospital, Sydney, NSW, Australia \\ ${ }^{6}$ Department of Ophthalmology Prince of Wales Hospital, University of New South Wales, Sydney, NSW, Australia
}

Rhinology 53: 290-302, 2015

DOI:10.4193/Rhino15.020

\begin{abstract}
Background: Intranasal corticosteroids (INCS) are prescribed for the long-term prophylactic treatment of inflammatory upper airway conditions. Although some systemic absorption can occur via topical routes, the clinical relevance is controversial. The effects of orally administered corticosteroids on intraocular pressure (IOP) and lens opacity (LO) are well established, but the impact of the INCS is less well defined. This study aims to systematically review the literature for evidence of adverse occular events with INCS use.
\end{abstract}

Methodology: A systematic review of literature from Medline and Embase databases (January 1974 to 21 st of November 2013) was performed. Using the PRISMA guidelines, all controlled clinical trials of patients using INCS, that reported original measures of IOP, LO, glaucoma or cataract incidences were included. Studies with adjuvant administration of oral, inhaled and intravenous steroids were excluded.

Results: 665 articles were retrieved with 137 were considered for full-text review. Of these, 116 (85\%) were literature reviews and two were case reports. 19 studies (10 RCTs, 1 case-control, 8 case series) were included for the qualitative review, of which 18 reported data on IOP and 10 on cataract/LO. None $(n=0)$ of the 10 RCT reporting data on glaucoma or IOP demonstrated changes in IOP compared to control. Also none $(n=0)$ of the 6 RCTs reporting cataract or lens opacity demonstrated changes compared to control.

Conclusion: Data from studies with low levels of bias, do not demonstrate a clinically relevant impact of INCS on neither ocular pressure, glaucoma, lens opacity nor cataract formation.

Key words: Intranasal steroids, Intraocular pressure, Glaucoma, Cataract, Chronic upper airway disease

\section{Introduction}

The intranasal corticosteroids (INCS) are prescribed in the management of upper airway inflammation. With their well-known anti-inflammatory properties, they provide relief for rhinitis symptoms, decongest airway oedema, restore nasal breathing, decrease mucus production and reduce the recurrence rates of upper airway inflammation ${ }^{(1,4-6)}$. Therefore, INCS are recommended in many evidence-based guidelines, for the management of upper airway disease ${ }^{(7,8)}$.

Adverse effects of intravenous, oral and inhaled steroids following systemic absorption are well established ${ }^{(1-3)}$. In recent years, the development of molecules designed to achieve potent, localized activity on nasal mucosa with minimal risk of systemic exposure. Patients are able to benefit from the therapeutic effects of the corticosteroids, while minimizing the use of steroid therapy via other routes ${ }^{(9)}$. 
In recent years there have been an increasing number of case reports and case series frequently citing INCS as a contributing factor to ocular adverse effects, challenging their role as a safer option ${ }^{(10-12)}$. Although it has long been recognised that a high dose inhaled corticosteroids can lead to systemic adverse effects, including the risk of developing ocular hypertension or open-angle glaucoma ${ }^{(1,13)}$, there has been little indication of such risk with intranasal administration. The ability of INCS to cause adverse systemic effects is mediated via nasal mucosa, or the gastrointestinal tract absorption. The newer generations of intranasal steroids are not only highly lipophilic, but also undergo rapid and extensive first-pass metabolism following ingestion; and therefore, have negligible systemic absorption from the latter route. Systemic bioavailability of drugs such as mometasone furoate or fluticasone propionate is as low as $0.1 \%$, and less than $2 \%$, respectively. This compares favorably to older generation of INCS, where one-third to half of an intranasal dose can reach the systemic circulation. Additionally, compared to inhaled corticosteroids, nasal steroids are usually administered in much smaller doses with more limited contribution to the systemic circulation ${ }^{(4,9,13,14)}$. Hence, the systemic effect of INCS, and its adverse clinical impact remain controversial.

In recent years, there have been published case reports and series, reporting changes in intraocular pressure or lens opacity in those consuming INCS ${ }^{(11-13)}$. However, few higher-level studies are cited when discussing ocular effects from INCS. Narrative reviews have the potential for bias in their summaries when not systematic in the review of the literature. Therefore, the aim of this study is to systematically review the evidence of the effect of INCS on potential adverse ocular effects.

\section{Materials and methods}

A systematic review was performed to identify published manuscripts with original data on any ocular effects from the use of INCS. PRISMA guidelines ${ }^{(23)}$ and recommendations from the Cochrane Handbook of Systematic reviews were followed where possible.

\section{Databases searched}

The electronic databases were used to conduct the search. Medline database was searched from 1946 to 21st of Nov 2013. Embase database was searched from 1974 to 22nd of Nov 2013. The bibliographies of studies subject to full-text analysis was reviewed and used as a further data source.

\section{Search strategy}

A search strategy was constructed using a combination of medical subject headings and keywords relevant to nasal and sinus disease as well as intranasal steroids. The search strategy used to search the electronic databases, Embase, and Medline databases

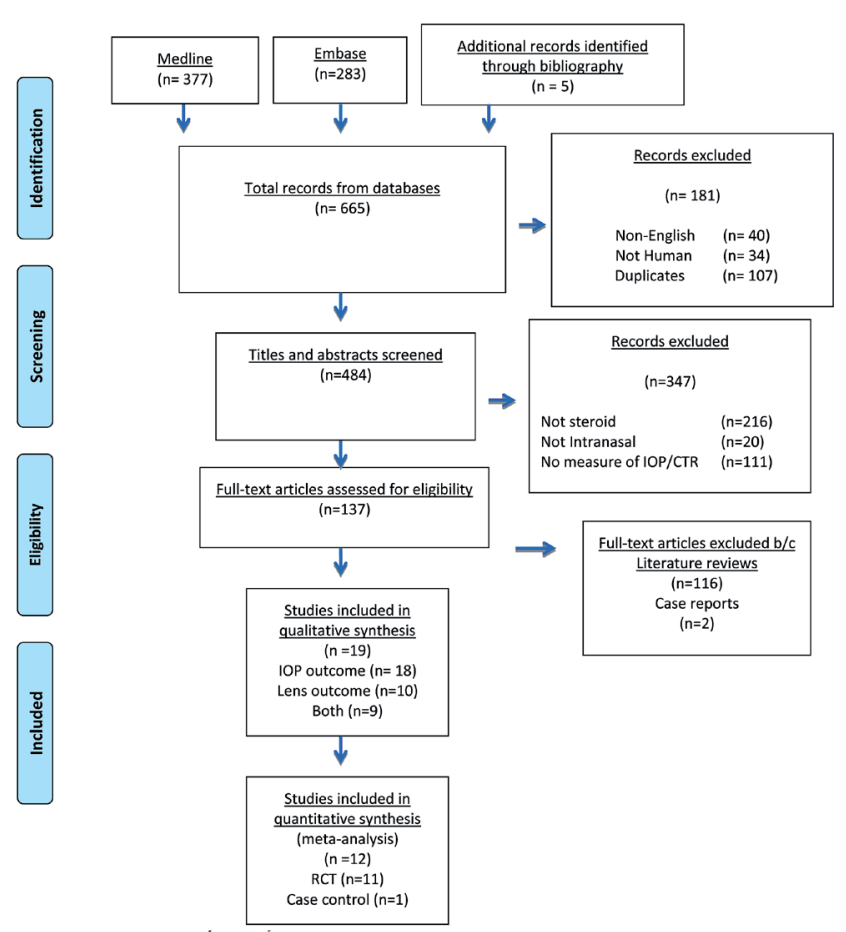

Figure 1. PRISMA chart.

are shown in Table 1.

\section{Study selection}

Study selection was performed by two authors (NA and RJH) in an unblended standardized manner. The following steps were taken. The identified publications from the electronic databases were combined. The lists of identified publications were scanned using PRISMA guidelines ${ }^{(15)}$, shown in Figure 1. Non-English, non-human studies, and duplicate publications were identified and removed.

The publications extracted were then further scanned using a combination of abstract or full-text. Those, which failed to use steroid as a treatment, apply it exclusively intranasal, nor reported original data of intraocular pressure or lens opacity were excluded.

The publications extracted were grouped by title review into studies that are case reports, literature reviews or comparative studies. The two former were excluded and the later was further included for qualitative synthesis.

\section{Criteria for included studies}

\section{Type of studies}

Any studies on population of patients using INCS were included. Randomized controlled trials (RCTs), cohorts, case-control and case series were caught. Simple case reports were not included. 


\section{Types of participants}

Adults and children with either, allergic rhinitis, (seasonal or perennial or moderate-severe persistent allergic rhinitis) confirmed by skin prick testing or in-vitro test for an allergen specific lgE or CRS as defined by either European Position Paper on Rhinosinusitis and Nasal polyps $2007^{(16)}$, or Rhinosinusitis Task Force Report ${ }^{(17)}$ and its revision ${ }^{(18)}$ were included.

\section{Types of interventions}

Studies involving topical steroid therapies versus either placebo, no treatment or another topical steroid therapy were considered. Trials using any co-interventions of oral steroid, antihistamines, decongestants, antibiotics (topical or intravenous) were included when the co-interventions were equally applied in both groups.

\section{Outcome measures}

All outcomes reporting changes or abnormality in IOP, visual acuity, cup to disc ratio, ocular hypertension, or the detection, and or alteration of known glaucoma were assessed. Additionally, any change relating to, cataract or measurements of lens opacity were also included.

\section{Exclusion criteria}

Any study that did not report an outcome of IOP, glaucoma assessment, lens opacity or cataract assessment. Case reports only. An exogenous corticosteroid taken either topically, inhaled or orally when not applied equally between controls and INCS users.

\section{Risk of bias}

Risk of bias in randomized controlled trials was assessed at a study level using the Cochrane Risk of Bias Assessment Tool (19).

\section{Statistical analysis}

Statistical analyses were performed using SPSS v 20.0 (Statistical Package for the Social Sciences, Chicago, IL, USA). Data was presented as descriptive statistics only. Parametric scale data was described with mean and standard deviation. Nonparametric data is described with median and interquartile range (IQR). Where, no IQR was provided, absolute range was recorded.

\section{Results}

The search produced a total of 660 studies. The first-level screening removed 107 irrelevant studies or duplicates, 40 nonEnglish, and 34 studies where the subjects were not human. The flowchart of the study selection using PRISMA flowchart ${ }^{(15)}$ is provided in Figure 1. All 479 remaining abstracts were reviewed to assess if they met the inclusion criteria. An additional 5 studies were identified from bibliographic search from reviewed articles, brining the total of the studies to 484 to be screen.
The title and abstract screen of the references identified 216 (44.6\%) studies that did not use corticosteroids, 20 (4.1\%) that either failed to apply the steroid intranasally or failed to avoid oral, inhaled or intravenous steroids as co-intervention, and $111(23.0 \%)$ of the studies that failed to report original data on intraocular pressure, lens opacity or incidence of glaucoma or cataract. The full text analysis of the remaining 137 studies identified 116 (84.7\%) studies that were literature reviews, and $2(1.5 \%)$ case reports. Nineteen $(n=19)(13.9 \%)$ studies were included.

\section{Included studies}

Of these 19 included studies, 9 reported data outcome on IOP $(3,6,11,13,20-24), 1$ on lens opacity ${ }^{(25)}$, and 9 studies reported data on both IOP and lens opacity ${ }^{(14,26-33)}$. This then left $18(94.7 \%)^{(3,6,11,}$ $13,14,20-33)$ studies on IOP, and $\left.10(55.6 \%)^{(14,25-33}\right)$ on lens opacity in clearly defined patient groups that could be used for comparison in this systematic review ${ }^{(14,26-33)}$. The study characteristics of the 19 included manuscripts are described in Tables 4 and 5. Studies followed the standard practices. The diagnosis of glaucoma was either based on the degree of increase in IOP $(>10 \mathrm{mmHg}$ or $>20 \%$ from the baseline) or the measure of IOP alone $(>20 \mathrm{mmHg})$. The same process was followed in measuring the lens opacity, whereby the diagnosis was made by a qualified ophthalmologist in accordance to a rigid set of guidelines. This involved slit lamp examination and refractometer.

\section{Intraocular pressure and INCS}

Of the 19 studies included in this review, 18 (94.7\%) reported outcomes on IOP, but only $11(63.2 \%)^{(1,20-23,26-31)}$ had a control group. These studies included 10 RCTs $(57.9 \%)^{(6,20-23,26-31)}$ and one case-control study ${ }^{(1)}$.

Of the eleven RCTs, three $(n=3)$ assessed children aged between 2 to 11 years old ${ }^{(28-30)}$, three $(n=3)$ on children $(>12$ years old) and adults $(26,27,31)$, and four $(n=4)$ on adults between 18 to 65 years of age ${ }^{(20-23)}$. The case control study included both children and adults population ${ }^{(1)}$. Majority of the comparative studies were conducted on individuals with Seasonal (SAR) or Perennial (PAR) Allergic Rhinitis for at least 6 months and positive skin prick test, who were exclusively being treated with no other form of delivery method of steroids other than intranasal. One study ${ }^{(22)}$ was conducted on postoperative FESS patients; and the case-control study ${ }^{(1,3)}$ was conducted on individuals with glaucoma and ocular hypertension who were also happen to be on intranasal corticosteroids. All studies except four ( $\mathrm{n}=$ 4) $(22,23,29,30)$. RCTs excluded individuals with glaucoma or ocular hypertension. The comparative studies included in this systematic review, together report on the effect of a variety of intranasal steroids including Fluticasone, Mometasone, Beclomethasone, Ciclesonide, and Budesonide. Almost all studies reported spe- 
Table 1. Search strategy used for Medline. A similar strategy was used in Embase.

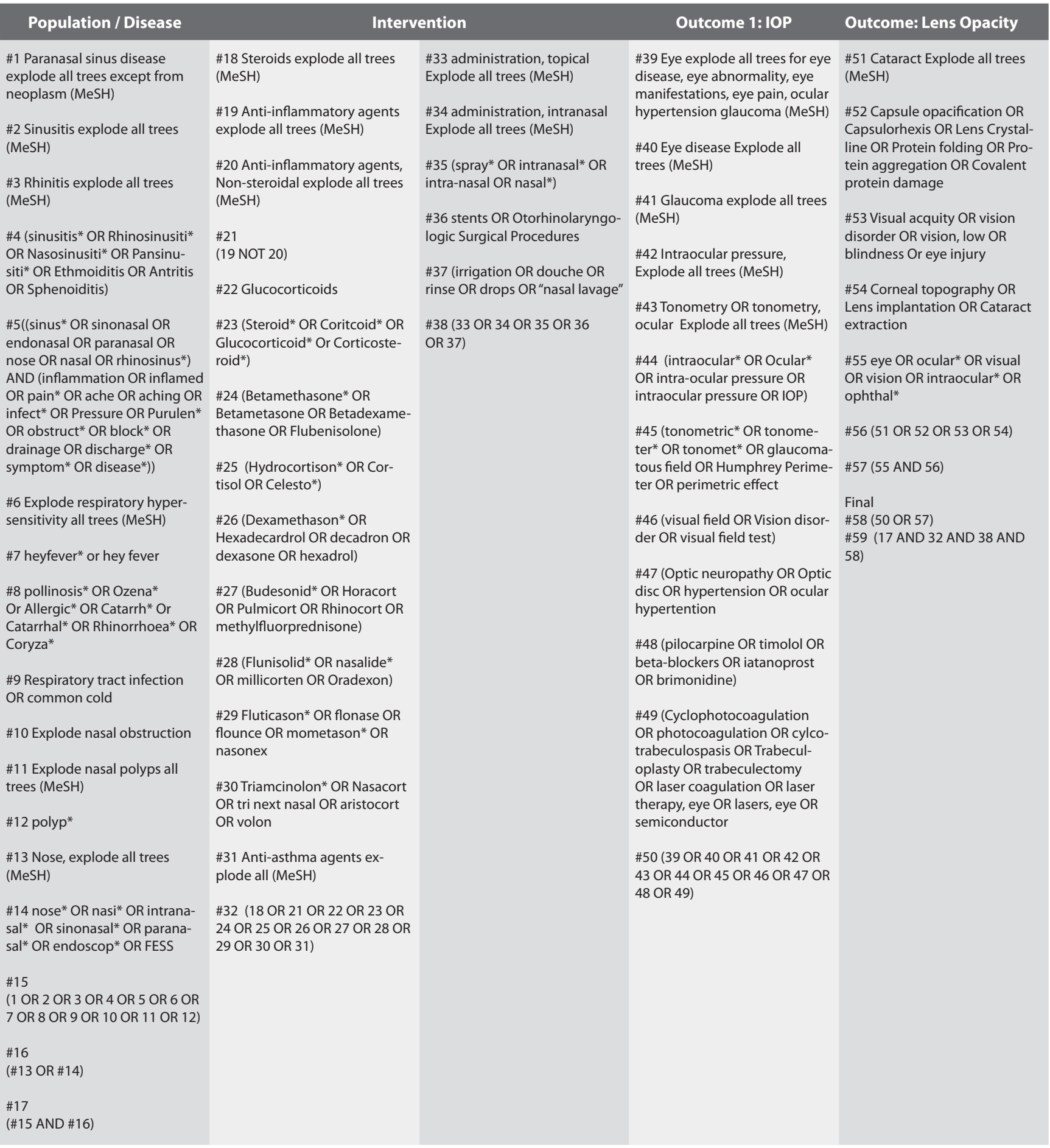

cific tools such as tonometry in addition to detailed physician examination in collecting the intraocular pressure measurements; and defined the measures considered abnormal prior to commencing the study. The outcomes were either reported as detailed measures of changes in IOP (continuous), and/or presence or absence of glaucoma (dichotomous).

The study outcomes for intraocular pressure and occurrence of glaucoma are described in Table 4. A total of 4376 patients in 10 RCTs were assessed for the effect of INCS on IOP and occurrence of glaucoma. Four studies report data on adults, three on children, and three on patients above twelve years of age. The studies assess a range of INCS. The most commonly used INCS was Fluticasone at doses ranging from 55 to $110 \mathrm{mcg}$ per day. All studies, except one, compare the INCS group with a placebo group. All studies measured the outcome with tonometry or 


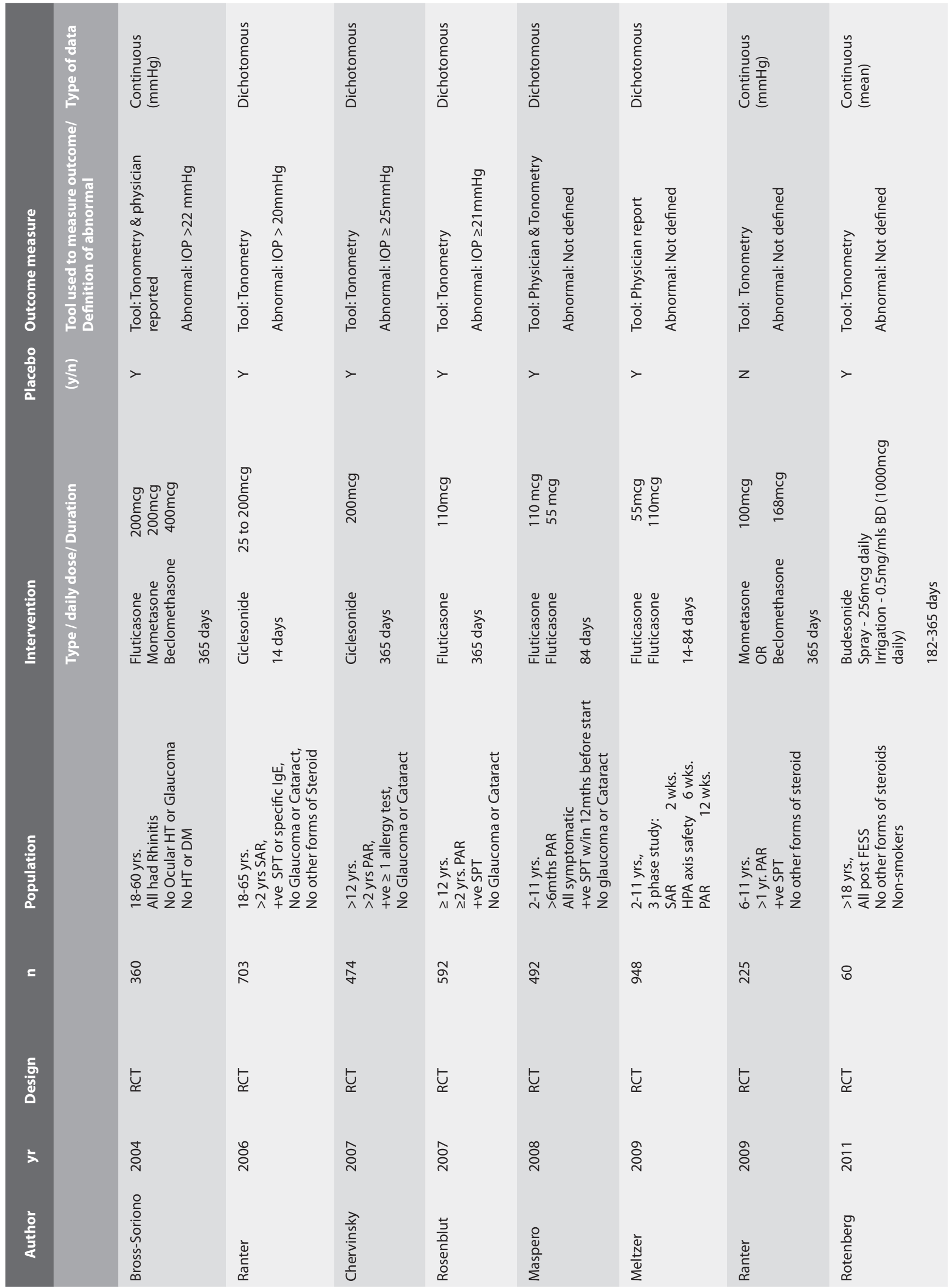


Table 2. Continued.
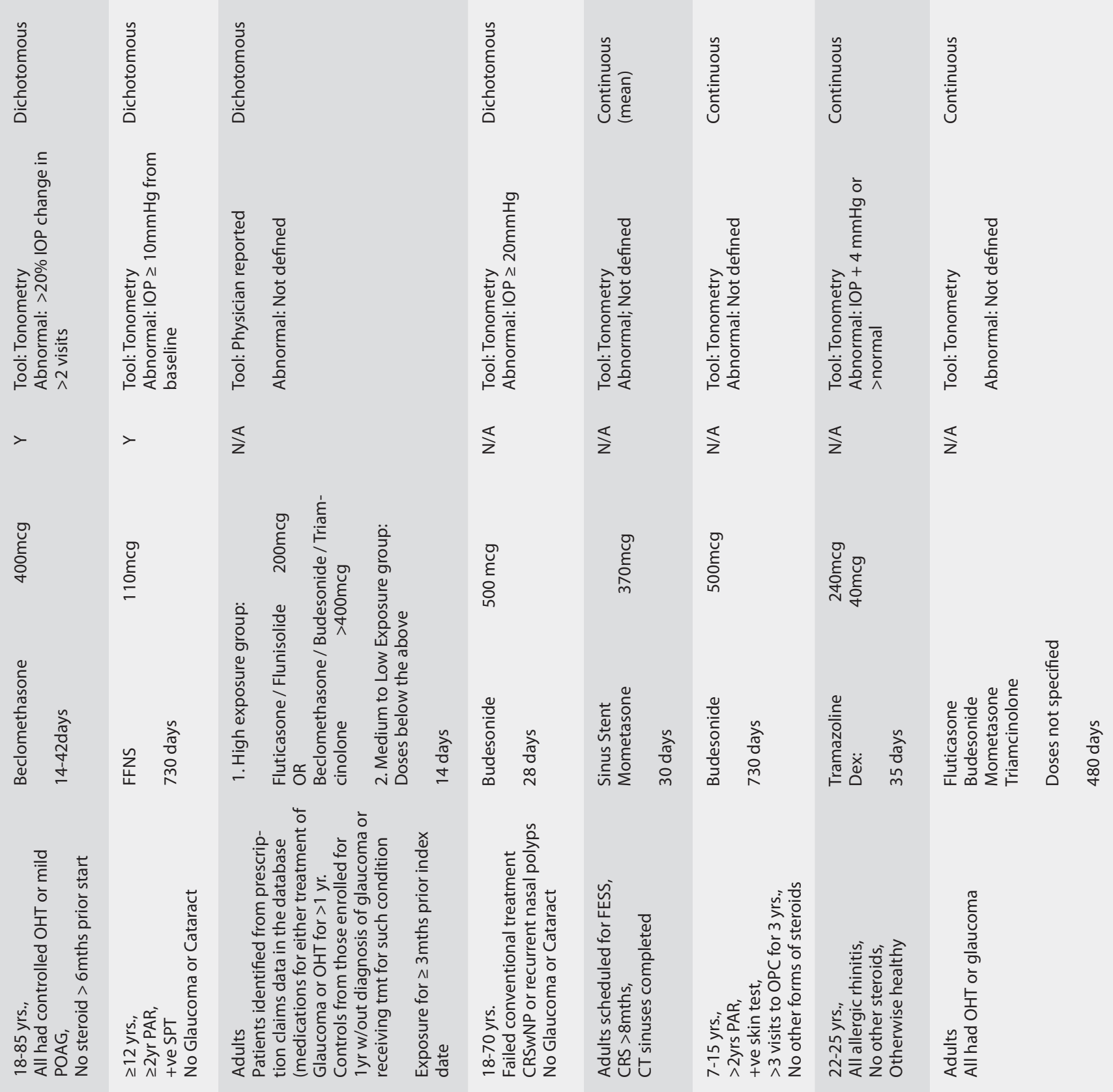

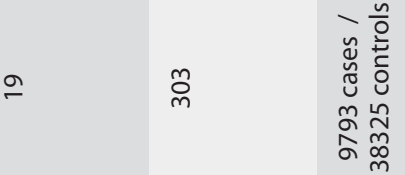
$\infty \quad$ in
옴
in

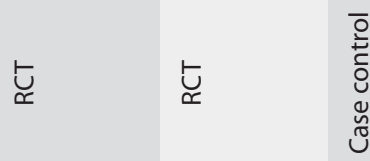

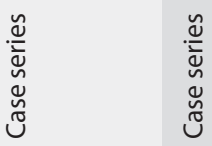

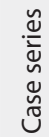

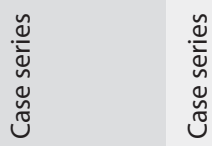
in $\quad \stackrel{m}{i}$ à
$\stackrel{m}{i} \quad \bar{i}$
휴
ڤ్రి

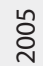

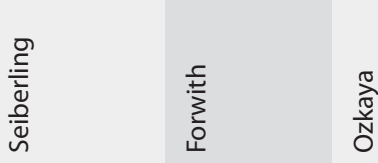

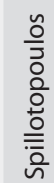
言 

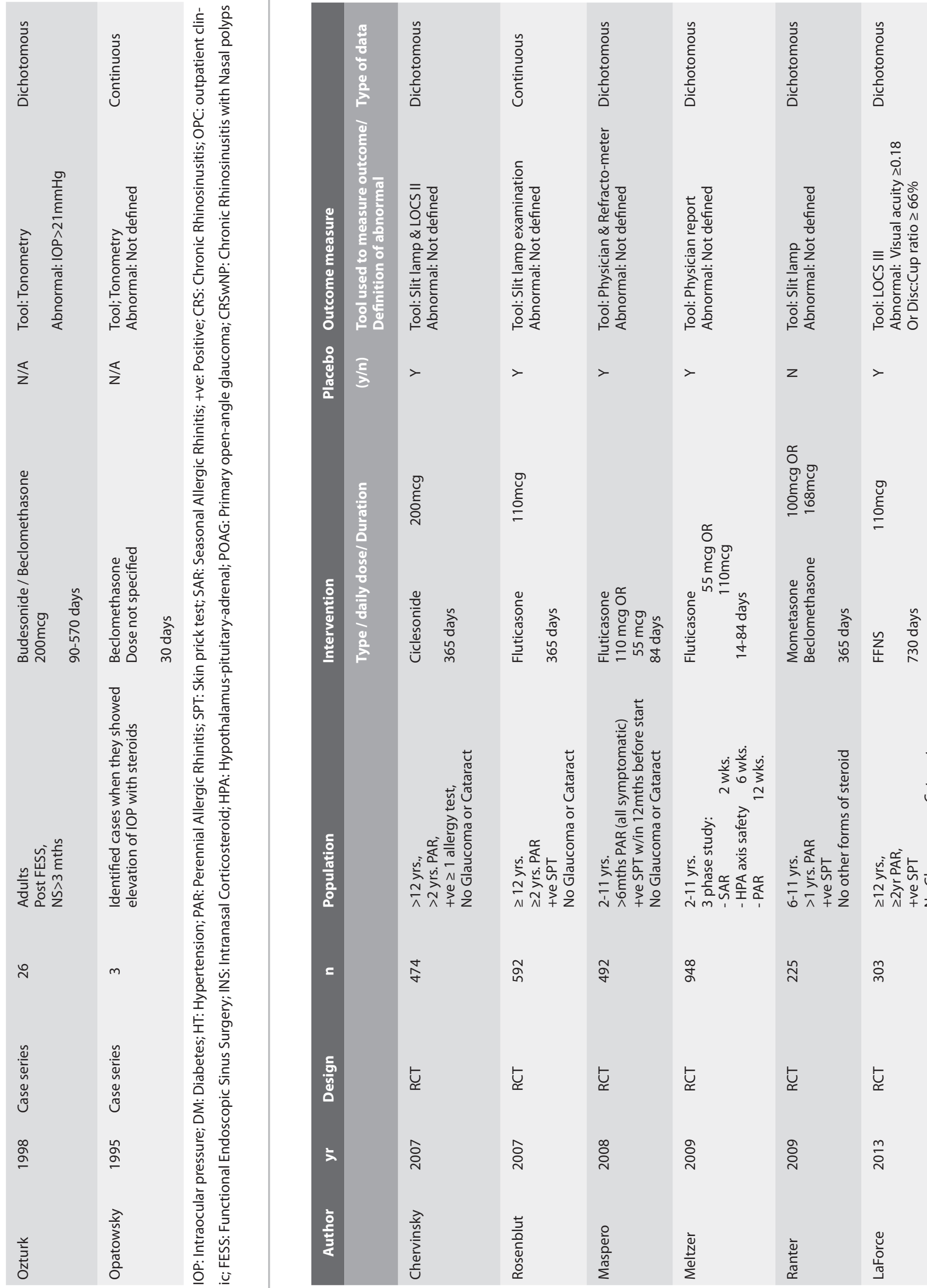
Table 3. Continued.

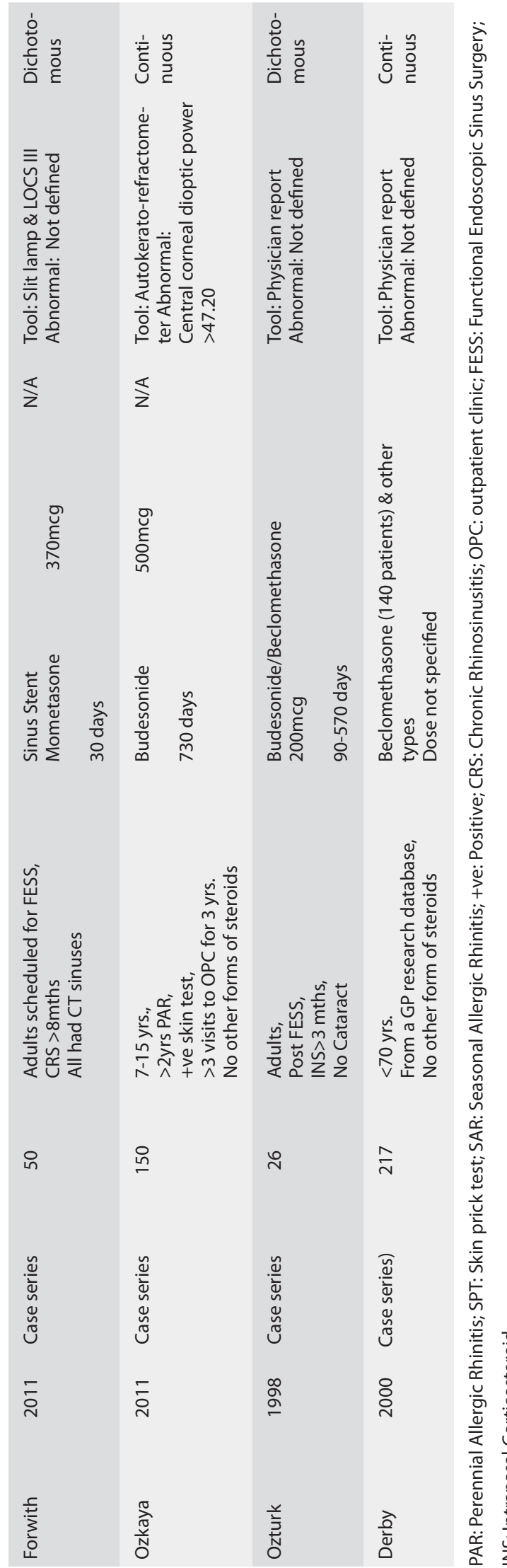

Table 4. Results from included studies with investigations into intra-occular pressure (IOP) or glaucoma incidence.

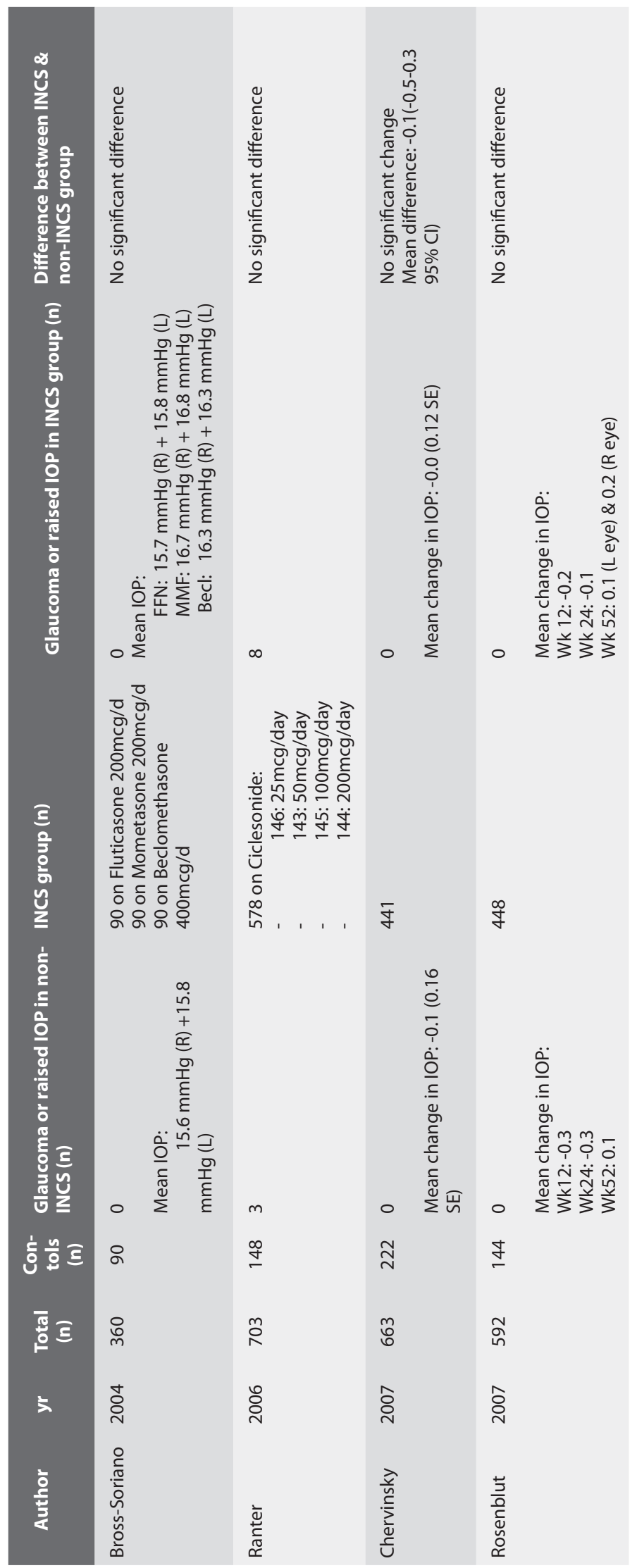


Table 4. Continued.

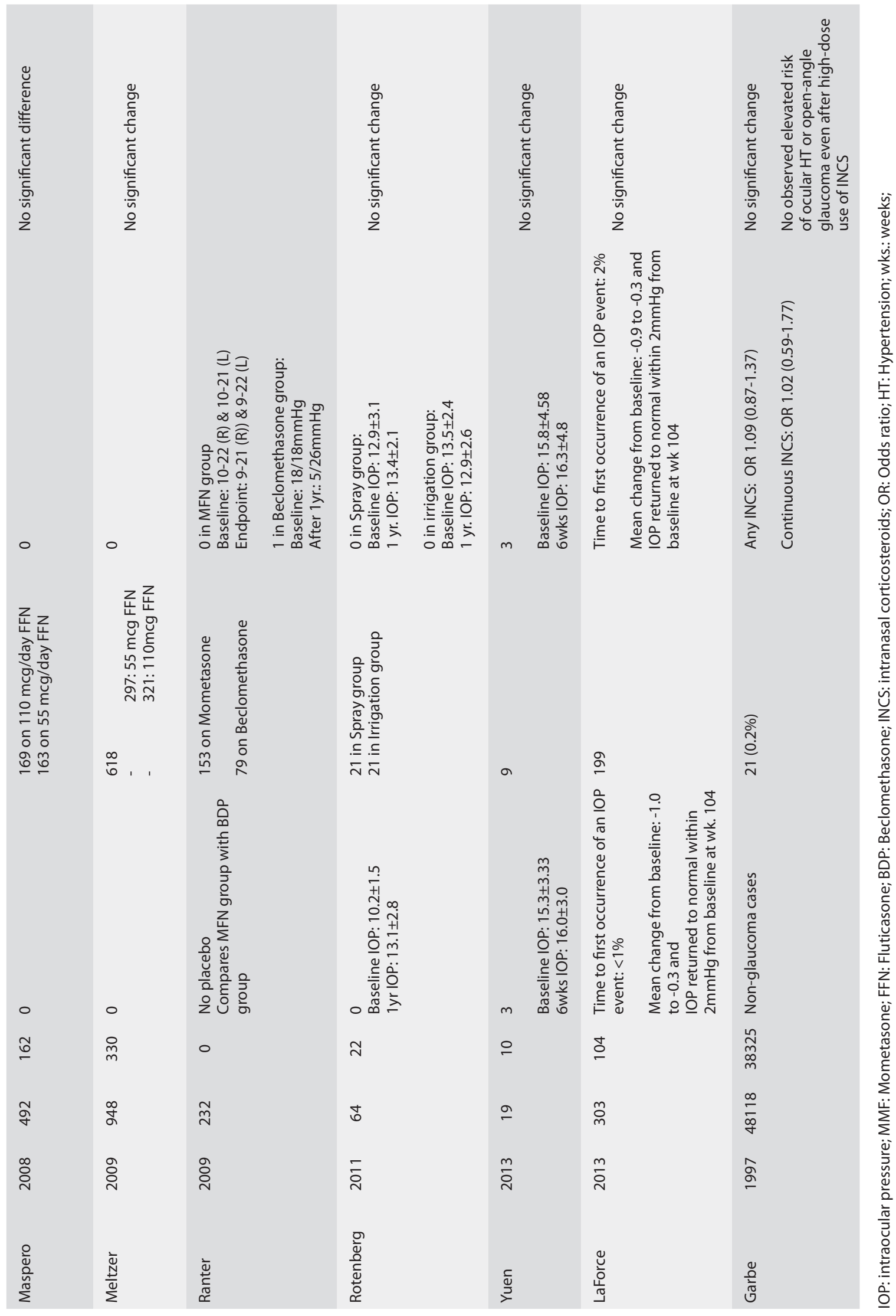


Table 5. Results of the included studies investigating lens opacity (LO) or the formation of cataracts.

\begin{tabular}{|c|c|c|c|c|c|c|c|}
\hline Author & yr & $\mathbf{n}$ & $\begin{array}{l}\text { Non-INCS } \\
\text { group }(n)\end{array}$ & $\begin{array}{l}\text { Cataract or } \\
\text { increase in lens } \\
\text { opacity in non- } \\
\text { INCS (n) }\end{array}$ & INCS group (n) & $\begin{array}{l}\text { Cataract or increase } \\
\text { in lens opacity in } \\
\text { INCS group ( } n \text { ) }\end{array}$ & $\begin{array}{l}\text { Difference between the } \\
\text { INCS \& non-INCS group }\end{array}$ \\
\hline Chervinsky & 2007 & 474 & 165 & $<1 \%$ & 331 & $<1 \%$ & No Significant difference \\
\hline Rosenblut & 2007 & 592 & 144 & $1(0.50 \%)(P S C)$ & 448 & $2(0.33 \%)(P S C)$ & No Significant difference \\
\hline Maspero & 2008 & 492 & 162 & 2 (PSC) & $\begin{array}{l}\text { 169: } 110 \mathrm{mcg} / \text { day FFN Group A } \\
\text { 163: } 55 \mathrm{mcg} / \text { day FFN Group B }\end{array}$ & $\begin{array}{l}0 \text { in group } A \\
1 \text { in group } B\end{array}$ & No Significant difference \\
\hline Meltzer & 2009 & 948 & 330 & 2 & $\begin{array}{ll}618 & \\
- & 297: 55 \mathrm{mcg} \text { FFN } \\
- & 321: 110 \mathrm{mcg} F F N\end{array}$ & 0 & No Significant difference \\
\hline Ranter & 2009 & 205 & $\begin{array}{l}\text { 68: BDP } \\
\text { (no placebo, } \\
\text { Compares } \\
\text { two groups } \\
\text { on INCS) }\end{array}$ & 1 & $\begin{array}{l}\text { 137: MFN } \\
\text { Compares two groups on INCS - } \\
\text { no placebo) }\end{array}$ & 0 & No Significant difference \\
\hline LaForce & 2013 & 303 & 104 & 0 & 199 & 0 & No Significant difference \\
\hline
\end{tabular}

PSC: Posterior subcapsular cataract

Table 6. Outcome of case reports and case series - intraocular pressure or lens opacity.

\begin{tabular}{lccccll} 
Author & yr & Type & Population $(\mathbf{n})$ & Focus & Outcome & Conclusion \\
\hline Opatowsky & 1995 & Case series & 3 & IOP & $\begin{array}{l}\text { 1 Cataract } \\
2 \text { IOP increase }\end{array}$ & INCS increase IOP and risk of cataract \\
\hline Bui & 2005 & Case series & 12 & IOP & $\begin{array}{l}\text { Mean change detected } \\
\text { But significance not measured }\end{array}$ & INCS can contribute to increase in IOP \\
\hline Perdis & 2010 & Case report & 1 & IOP & $\begin{array}{l}\text { Withdrawal from steroid, } \\
\text { reduced IOP }\end{array}$ & Withdrawal from INCS reduces IOP \\
\hline Lui & 2011 & Case report & 1 & CTR & Cataract & INCS increase risk of cataract \\
\hline
\end{tabular}

objective standard tools by a physician trained to examine the eye. The selected abnormal measures for IOP range from $>21$ to $>25 \mathrm{mmHg}$. Almost all outcomes reported were dichotomous. And all studies, including the studies that did not exclude patient with pre existing intraocular hypertension or glaucoma $(3,22,23,29,30)$, reported no significant additional increase in IOP or occurrence of glaucoma in the INCS arm.

\section{Lens opacity and INCS}

Ten out of the $19(52.6 \%)^{(14,25-33)}$ studies included for qualitative analysis reported outcome on lens opacity, but only 6 (31.6\%) ${ }^{(26-31)}$ studies were randomised control studies. In contrast to the studies reporting on IOP, all studies included children in their selected population. Three studies included patients above twelve years of age $(26,27,31)$, and three $(n=3)$ included children between the ages of two to eleven ${ }^{(28-30)}$. In all studies, the patient population suffered from PAR or SAR for at least 6 months and had a positive skin prick test. All studies excluded patients with known glaucoma or cataract. The studies were conducted for minimum of 6 weeks to a maximum of 2 years. In contrast to the studies on IOP outcomes, studies on lens opacity were limited to assessing the effects of Fluticasone or Ciclesonide and their relation to presence of cataract (in particular subcapsular cataract).

The outcomes for changes in lens opacity and occurrence of cataract are described in Table 5. The table demonstrates a total of 3014 patients in 6 RCTs. Half of the studies reported exclusively on children, and the other half on both adults and children above the age of twelve years. All patients were diagnosed with PAR for at least six months, and had at least one positive skin prick test. The most commonly tested INCS was Fluticasone at doses of 55 to $110 \mathrm{mcg}$ per day. The patients underwent slit lamp examination by an ophthalmologist. All outcomes except in one study were dichotomous. Two studies reported the same 
Table 7. Risk of bias.

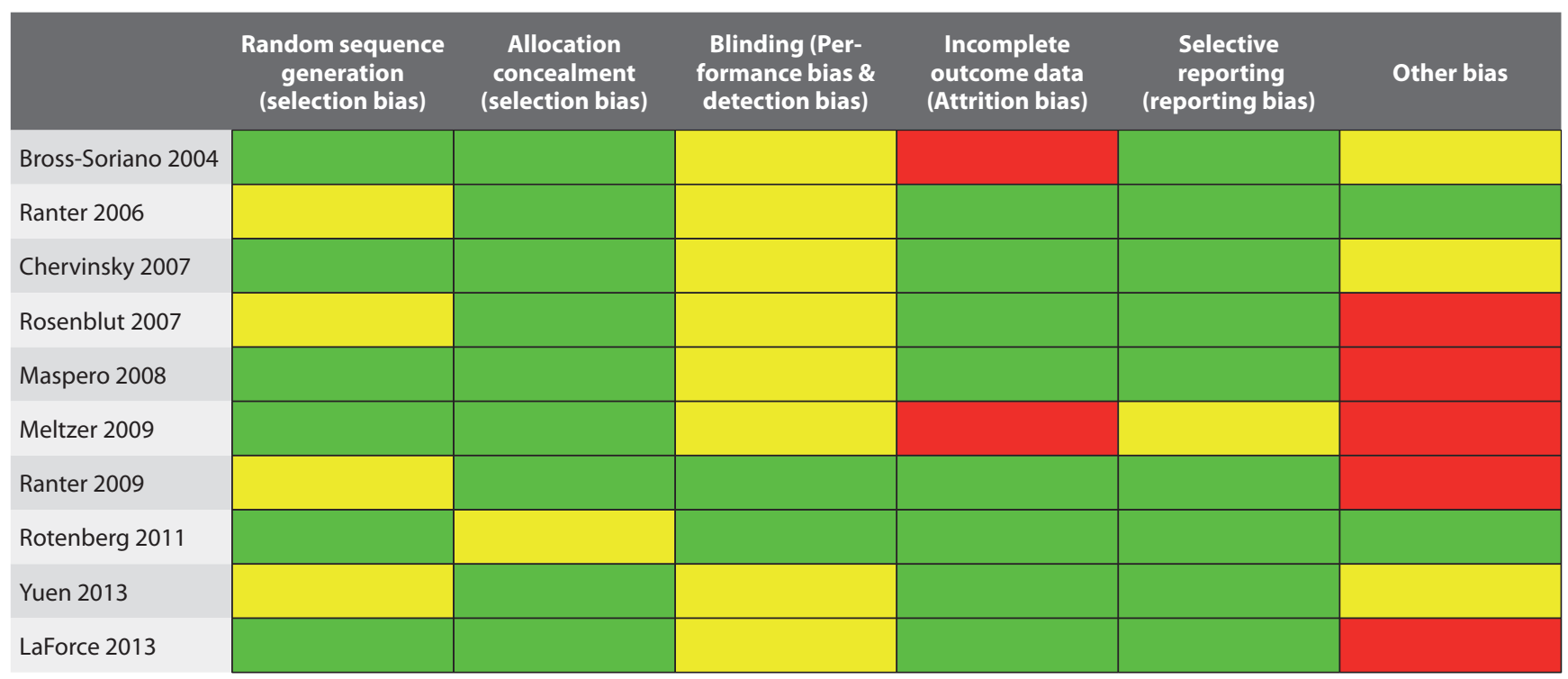

green: low risk of bias; yellow: unclear risk of bias; red: high risk of bias

rate of cataract detected in the placebo group; while all other studies reported zero outcomes for cataract in the INCS groups.

\section{Risk of bias}

Comprehensive reporting of study methodology was inconsistent amongst randomised control trials (RCTs) (Table 7). Sequence generation randomization was reported but detail of sequence generation given in $50 \%(20,21,23,27,30)$. All studies reported blind evaluators but only two reported details of methods implemented to ensure blinding ${ }^{(22,30)}$. One study was open to patients ${ }^{(30)}$, however this study attempted to exclude patient bias by assessing evaluator outcomes only. All studies except one ${ }^{(22)}$, reported on concealment and allocation. Complete outcome data was reported in all but one ${ }^{(29)}$. Two studies ${ }^{(20,29)}$ failed to report outcome data on withdrawals. Half of the studies were sponsored by pharmaceutical companies ${ }^{(27-31)}$ with remaining studies did not report any conflict of interest.

\section{Discussion}

The changes in lens opacity and ocular hypertension from corticosteroids, administered orally, intravenous and, to a lesser degree, inhaled, are well documented. Prolonged exposure to the corticosteroids via any of these routes can result in intraocular pressure related visual loss or cataract ${ }^{(12,25,29,33,34)}$. The adverse profile of the corticosteroids, results largely from systemic absorption of the drug regardless of delivery type ${ }^{(4)}$.

However, the occurrence of similar adverse events from intranasal delivery of the steroids remains controversial ${ }^{(12,25,33)}$. The literature is replete with non-systematic reviews on the subject ( $n=116)$ with $42 \%(n=8)$ of all original study representing case series only. In recent years, a rising trend of case reports and narrative literature reviews warn of the potential of INCS to cause ocular hypertension, worsening of preexisting glaucoma and changes in lens opacity, however, few offer more than level 4/5 data to support their recommendations. Such comments and case series/report data can lead to and environment of fear and subsequent poor prescribing patterns of intranasal steroids by primary care physicians ${ }^{(10-13,29,36)}$.

The established success of INCS in treatment of upper airway inflammatory disease has led to increased long term prescribing patterns by specialists and recommendations for long-term use $(16,18)$. The intranasal formulations are designed to allow direct local delivery of the drug to the target area, whilst minimizing the risk of systemic absorption ${ }^{(4,29)}$. The latest generation of INCS, such as Fluticasone propionate, Mometasone furoate, $\mathrm{Ci}$ clesonide, and Fluticasone furoate come close to the ideal INCS, in terms of the pharmacokinetic or pharmacodynamics properties. They have a high degree of glucocorticoid receptor affinity, potency and specificity; as well as low systemic bioavailability, high rate of hepatic first-pass metabolism and therefore rapid systemic elimination. Moreover, the slow-release drug-lipid complexes allow clinically desirable outcome with once-daily dosing ${ }^{(9,37)}$. This is all, in addition to remaining very successful in achieving the clinical efficiency by reducing mucosal swelling, improving sinus drainage and therefore hastening elimination of pathogens, and inhibiting the release and formation of mucous secretogogues $(4,5,25,33)$.

In 1960, Black et al. ${ }^{(38)}$, first described the association between systemic corticosteroids and the development of posterior 
subcapsular cataract in patients with rheumatoid disease.

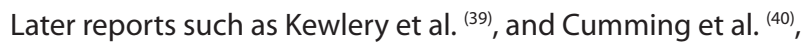
confirmed similar association with the use of inhaled corticosteroids. However, intranasal steroids do vary significantly in their systemic absorption, bioavailability, and lipophilicity, to the corticosteroids administered via other routes ${ }^{(4)}$. With intranasal delivery, up to $30 \%$ of the dose is deposited and remain in the nose depending on the drug's lipophilicity; the remainder is swallowed. Ninety-nine percent of the swallowed INCS is subject to the first-pass metabolism in the liver for second-generation agents. Hence, the systemic side effect from INCS depends on not only the lipophilicity of the administered corticosteroid and direct absorption via the nasal mucosa, but also its systemic and local bioavailability, and hepatic first-pass metabolism ${ }^{(4,9,13,25 \text {, }}$ 36,37). Whereas, inhaled corticosteroids, which have been linked to systemic adverse events, are primarily absorbed through the respiratory mucosa of the lower airway. And although, oropharyngeal deposition may occur, only a fraction is swallowed and undergoes hepatic first-pass metabolism. The remainder is absorbed systemically ${ }^{(9,37,41)}$. Doses used in the lower airway are traditionally much larger than those used intranasally.

Fraunfelder et al. (36), argues that nasal steroids can reach ocular structures in levels sufficient to cause ocular hypertension and posterior subcapsular cataract in susceptible patients. This study had identified 21 patients who had PSC following administration of nasal or inhaled Beclomethasone. Most of the patients were on the medication for more than five years and often in higher than recommended dose of $252 \mathrm{mcg} /$ day. Most importantly, 9 out of 21 patients were also using systemic steroids concomitantly. The concomitant use of systemic steroids in a large number of the patients, in these studies, is a significant confounding factor. Eight years later, Ozturk et al. ${ }^{(14)}$, demonstrated a lack of any considerable change in the lens opacity of all patients studied, despite using higher dose of nasal Beclomethasone (400 $\mathrm{mcg} /$ day) for over 19 months.

This systematic review found no difference in intraocular pressure or diagnosis of glaucoma in 4376 patients across 10 randomised control trials ${ }^{(20-23,26-31)}$, exposed to a range of intranasal steroids (including new and old generation INCS) from 2-104 weeks, between controls and INCS users. Therefore, level one evidence supports a low risk for INCS use in the management of upper airway inflammatory disease. With a varying duration of INCS from 2 weeks to 2 years, IOP changes can occur within these time frames. IOP changes can be seen within hours after a parenteral dose of corticosteroid ${ }^{(42)}$ and current recommenda- tions within the ophthalmic literature suggests a 2 week review of IOP when patients commence non-parenteral exogenous corticosteroid use ${ }^{(43)}$. There were 5 studies that included patients with glaucoma or ocular hypertension in their analysis. Four of these studies were RCTs ${ }^{(22,23,29,30)}$ and one case control ${ }^{(1)}$. All five studies concluded that INCS does not worsen existing ocular hypertension or glaucoma.

This review also found that there was no difference in lens opacification or the new diagnosis of cataracts between INCS users and control group in 3014 patients across 6 randomised control trials (26-31), following exposure to Ciclesonide or Fluticasone for 6 to 104 weeks. Therefore, level one evidence supports INCS as a safe treatment of upper airway inflammatory disease, and does not appreciably increase the risk of cataract in patients exposed to this medication for a considerably long period of time.

Meta-analysis of the data was not possible, as almost all studies reviewed in this systematic review, reported zero adverse ocular outcomes. This is an obvious limitation of our study as the zero adverse effects can either represent the absence of a true effect or an underpowered study, however the risk is likely to be very small.

\section{Conclusion}

Data from studies with low levels of bias, do not demonstrate a clinically relevant impact of INCS on neither ocular pressure, glaucoma, lens opacity nor cataract formation Level 1 studies report that INCS can be used without fear of ocular side effects resulting from INCS therapy. And even, in the presence of preexisting ocular hypertension, level one data suggests negligible impact from INCS therapy.

\section{Acknowledgement}

Ellie Pratt and Nadiene Mrad (Otolaryngology and Skull Base Surgery, Applied Medical Research Centre, St Vincent Hospital, Sydney, NSW, Australia)

\section{Authorship contribution}

NA: Data collection, analysis and manuscript production; KS: Data collection and analysis. LK: Manuscript review and data analysis; RS: Design and expert manuscript review; KT: Manuscript review and preparation; GW: Manuscript review and preparation; $\mathrm{RH}$ : Design, analysis and manuscript review.

\section{Conflict of Interest}

The authors decelare no conflict of interest.

\section{References}

1. Garbe E, LeLorier J, Boivin J-F, Suissa S
Inhaled and nasal glucocorticoids and the risks of ocular hypertension or open-angle glaucoma. JAMA. 1997; 277(9):722-7.

2. Barnes N. Safety of high-dose inhaled corti- 
costeroids. Resp Med. 1993;87:27-31.

3. Garbe E, LeLorier J, Boivin J-F, Suissa S. Risk of ocular hypertension or open-angle glaucoma in elderly patients on oral glucocorticoids. Lancet. 1997;350(9083):979-82.

4. Demoly P. Safety of intranasal corticosteroids in acute rhinosinusitis. Am Otolaryngol. 2008;29(6):403-13.

5. Bonfils $P$, Norès JM, Halimi $P$, Avan P Corticosteroid Treatment in Nasal Polyposis With a Three-Year Follow-Up Period. Laryngoscope. 2003;113(4):683-7.

6. Seiberling KA, Chang DF, Nyirady J, Park $F$, Church CA, editors. Effect of intranasal budesonide irrigations on intraocular pressure. Int Forum Allergy Rhinol. 2013:3(12):953-7.

7. Fokkens WJ, Lund VJ, Mullol J, et al. EPOS 2012: European position paper on rhinosinusitis and nasal polyps 2012. A summary for otorhinolaryngologists. Rhinology 2012;50(1):1-12

8. Rudmik L, Hoy M, Schlosser RJ, et al., editors. Topical therapies in the management of chronic rhinosinusitis: an evidence-based review with recommendations. Int Forum Allergy Rhinol. 2013:3(4):281-98.

9. Derendorf H, Meltzer EO. Molecular and clinical pharmacology of intranasal corticosteroids: clinical and therapeutic implications. Allergy. 2008:63(10):1292-300.

10. Bielory L, Blaiss M, Fineman SM, et al. Concerns about intranasal corticosteroids for over-the-counter use: position statement of the Joint Task Force for the American Academy of Allergy, Asthma and Immunology and the American College of Allergy, Asthma and Immunology. Ann Allergy Asthma Immunol. 2006 Apr;96(4):514-25.

11. Bui CM, Chen $H$, Shyr $Y$, Joos KM Discontinuing nasal steroids might lower intraocular pressure in glaucoma. J Allergy Clin Immunol. 2005;116(5):1042-7

12. Liu A, Manche EE. Bilateral posterior subcapsular cataracts associated with longterm intranasal steroid use. J Cataract Refract Surg. 2011;37(8):1555-8.

13. Opatowsky I, Feldman RM, Gross R, Feldman ST. Intraocular pressure elevation associated with inhalation and nasal corticosteroids. Ophthalmology. 1995:102(2):177-9.

14. Oztürk $F$, Yücetürk $A$, Kurt $E$, Unlü $H$, Ilker $S$. Evaluation of intraocular pressure and cataract formation following the long-term use of nasal corticosteroids. Ear, Nose, Throat J. 1998;77(10):846-8, 50-1.

15. Moher D, Liberati A, Tetzlaff J, Altman DG. Preferred reporting items for systematic reviews and meta-analyses: the PRISMA statement. Ann Int Med. 2009;151(4):264-9.

16. Fokkens WJ, Lund VJ, Mullol J. European position paper on rhinosinusitis and nasal polyps 2007. Rhinology Supplement. 2006 (20):1-136.

17. Benninger MS, Ferguson BJ, Hadley JA et al. Adult chronic rhinosinusitis: definitions, diagnosis, epidemiology, and patho- physiology. Otolaryngol Head Neck Surg. 2003;129(3):S1-S32.

18. Snidvongs K, Heller GZ, Sacks R, Harvey RJ. Validity of European Position Paper on Rhinosinusitis Disease Control Assessment and Modifications in Chronic Rhinosinusitis. Otolaryngol Head Neck Surg. 2013:0194599813517080.

19. Higgins J, Altman DG, Gøtzsche PC, et al. The Cochrane Collaboration's tool for assessing risk of bias in randomised trials. BMJ. 2011;343.

20. Bross-Soriano D, Hanenberg-Milver C Schimelmitz-Idi J, Arrieta-Gomez JR, Astorga Del Toro R, Bravo-Escobar G. Effects of three nasal topical steroids in the intraocular pressure compartment. Otolaryngol Head Neck Surg. 2004;130(2):187-91.

21. Ratner $\mathrm{PH}$, Wingertzahn MA, van Bavel JH, et al. Effectiveness of ciclesonide nasal spray in the treatment of seasonal allergic rhinitis. Ann Allergy Asthma Immunol. 2006;97(5):657-63.

22. Rotenberg BW, Zhang I, Arra I, Payton KB. Postoperative care for Samter's triad patients undergoing endoscopic sinus surgery: A double-blinded, randomized controlled trial. Laryngoscope. 2011;121(12):2702-5.

23. Yuen D, Buys YM, Jin Y-P, Alasbali T, Trope GE. Effect of beclomethasone nasal spray on intraocular pressure in ocular hypertension or controlled glaucoma. J Glaucoma. 2013;22(2):84-7.

24. Spiliotopoulos C, Mastronikolis NS Petropoulos IK, Mela EK, Goumas PD, Gartaganis SP. The effect of nasal steroid administration on intraocular pressure. Ear Nose Throat J. 2007;86(7):394.

25. Derby L, Maier WC. Risk of cataract among users of intranasal corticosteroids. J Allergy Clin Immunol. 2000;105(5):912-6.

26. Chervinsky $P$, Kunjibettu $S$, Miller DL, Prenner BM, Raphael G, Hall N, et al. Long-term safety and efficacy of intranasal ciclesonide in adult and adolescent patients with perennial allergic rhinitis. Ann Allergy Asthma Immunol. 2007;99(1):69-76.

27. Rosenblut A, Bardin P, Muller B, et al. Longterm safety of fluticasone furoate nasal spray in adults and adolescents with perennial allergic rhinitis. Allergy. 2007;62(9):10717

28. Máspero JF, Rosenblut A, Finn Jr A, Lim J, Wu W, Philpot E. Safety and efficacy of fluticasone furoate in pediatric patients with perennial allergic rhinitis. Otolaryngol-Head Neck Surg. 2008;138(1):30-7.

29. Meltzer EO, Tripathy I, Máspero JF, Wu W, Philpot E. Safety and Tolerability of Fluticasone Furoate Nasal Spray Once Daily in Paediatric Patients Aged 6-11 Years with Allergic Rhinitis. Clin Drug Investig. 2009;29(2):79-86.

30. Ratner PH, Meltzer EO, Teper A. Mometasone furoate nasal spray is safe and effective for 1-year treatment of children with perennial allergic rhinitis. Int J Pediat
Otorhinolaryngol. 2009;73(5):651-7

31. LaForce C, Journeay GE, Miller SD, et al. Ocular safety of fluticasone furoate nasal spray in patients with perennial allergic rhinitis: a 2-year study. Ann Allergy Asthma Immunol. 2013;111(1):45-50.

32. Forwith KD, Chandra RK, Yun PT, Miller SK, Jampel HD. ADVANCE: A multisite trial of bioabsorbable steroid-eluting sinus implants. Laryngoscope. 2011;121(11):247380.

33. Ozkaya E, Ozsutcu M, Mete F. Lack of ocular side effects after 2 years of topical steroids for allergic rhinitis. J Pediatr Ophthalmol Strabismus. 2010;48(5):311-7.

34. Brookes $J$, Khaw PT. Steroid response in children. Clin Experiment Ophthalmol. 2005:33(3):229-30.

35. Knutsson U, Stierna P, Marcus C, CarlstedtDuke J, Carlström K, Brönnegård M. Effects of intranasal glucocorticoids on endogenous glucocorticoid peripheral and central function. J Endocrinol. 1995;144(2):301-10.

36. Fraunfelder F, Meyer S. Posterior subcapsular cataracts associated with nasal or inhalation corticosteroids. Am J Ophthalmol. 1990;109(4):489.

37. Trangsrud AJ, Whitaker AL, Small RE. Intranasal corticosteroids for allergic rhinitis. Pharmacotherapy. 2002;22(11):1458-67.

38. Black RL, Oglesby RB, von Sallmann L, Bunim JJ. Posterior subcapsular cataracts induced by corticosteroids in patients with rheumatoid arthritis. JAMA. 1960;174(2):166-71.

39. Kewley GD. Possible association between beclomethasone diproprionate aerosol and cataracts. J Paediat Child Health. 1980;16(2):117-8.

40. Cumming RG, Mitchell P, Leeder SR. Use of inhaled corticosteroids and the risk of cataracts. N Engl J Med. 1997;337(1):8-14.

41. Ryrfeldt $\AA$, Andersson P, Edsbäcker S, Tönnesson M, Davies D, Pauwels R. Pharmacokinetics and metabolism of budesonide, a selective glucocorticoid. Eur J Resp Dis Suppl. 1981;122:86-95.

42. Weinreb RN, Polansky JR, Kramer SG, Baxter JD. Acute effects of dexamethasone on intraocular pressure in glaucoma. Invest Ophthalmol Vis Sci. 1985;26(2):170-5.

43. Kersey JP, Broadway DC. Corticosteroidinduced glaucoma: a review of the literature. Eye. 2006;20(4):407-16.

\section{Nazanin Ahmadi \\ ENT department/Clinic \\ Hornsby Hospital \\ Palmerston $\mathrm{Rd}$ \\ Hornsby \\ NSW}

Australia, 2077 